\title{
Brain Storm Optimization for Sensors' Duty Cycle in Wireless Sensor Network
}

\author{
Ahmed Y Khedr ${ }^{1,2}$ \\ Systems and Computers Department, Alazhar University, Cairo, Egypt ${ }^{1}$ \\ Computer Science and Engineering, Hail University, Hail KSA ${ }^{2}$
}

\begin{abstract}
Wireless Sensor Networks (WSNs) became one of the emergent networks that are used in many real life applications. They are also used in many of the critical applications such as battle field monitoring and health care. Therefore, sensor nodes are required to survive for long time. However, such nodes suffer from low memory, limited storage, limiting sensing and communication ranges, limited energy sources, and limited processing capabilities. One of the effective methods to save the nodes' energy is the duty cycling where some of the nodes can go to sleep while others can perform the required monitored field tasks. In this paper, we propose a new algorithm that utilizes the concept of the people thinking which the Brain Storming Optimization (BSO) for sensor nodes duty cycle management. Through extensive set of experiments using homogenous and heterogeneous WSNs, BSO seems to outperform the Non-duty cycle algorithm.
\end{abstract}

Keywords: Brain Storming Optimization (BSO), Duty Cycle, energy saving, Wireless sensor networks.

\section{INTRODUCTION}

Duty-cycling has been considered as one of the most important techniques for energy conservation in wireless sensor networks. The duty-cycled WSNs enables nodes to stay dormant most of the time to save their energy dissipation and just be awake when sending or receiving packets [1]. Duty-cycled WSNs can be classified into two categories: synchronous and asynchronous. In synchronous duty-cycled WSNs, each node can synchronize their active and sleep periods with its neighbours which causes extra overhead. In asynchronous [2] duty cycled WSNs, nodes has theirown duty cycle schedule and operate independently of its neighbours.

Delay-aware Energy-optimized Flooding algorithm (DEF) is proposed in [4], by constructing delay-constrained energy optimal flooding trees in synchronous duty-cycled WSNs with unreliable links to improve energy efficiency and adjust the structure of a constructed flooding tree. [5] investigates multiple task scheduling problems for low-duty-cycled sensor networks. This work focuses on load balance and provides two efficient algorithms: scheduling greedy algorithm and heuristic algorithm to reduce congestion and data loss.

Geographic routing oriented Sleep Scheduling (GSS) is proposed in [6] to improve the first transmission path's performance of two-phase geographic greedy forwarding (TPGF) based connected-k neighbourhood (CKN) algorithm in duty-cycled WSNs. Latency Aware Broadcast Scheduling (LABS) algorithm is presented in [7] to solve the minimum latency broadcast scheduling problem. LABS algorithm constructs a shortest path tree using Dijkstra algorithm. Then, all sensor nodes are divided into different layers according to the distance to the root of the tree. LABS algorithm consists of two phases: broadcast-tree construction phase and scheduling phase, such that all the nodes can receive the broadcast message without collision.

[14] considers minimum latency aggregation scheduling (MLAS) problem that finds the fastest collision free schedule for data aggregation. It also proposes a Delay Efficient Data Aggregation Scheduling (DEDAS) scheme to generate a collision-free schedule and minimize the delay for data aggregation in duty-cycled WSNs. In the DEDAS scheme, data aggregation tree is created first and collision-free schedule for data aggregation is generated. Improved version (DEDAS-B) is proposed also in [14] to reduce the weakness of DEDAS in high density networks.

[15] speeds up data aggregation process by constructing a routing tree with smaller number of children for each inner node based on connected dominator set (CDS) with removing redundant connectors. To minimize total delay time two algorithms proposed in [15], the first one is a centralized Greedy Aggregation Scheduling (GAS) algorithm and the second named distributed Partitioned Aggregation Scheduling (PAS) algorithm. GAS is used to minimize the aggregation time while PAS is used to ensure the largest extent of channel reuse across space and time domains.

An On-demand Scheduling Cooperative MAC protocol (OSC-MAC) is proposed in [8] to solve the energy hole problem in multi-hop WSNs by using pipelined and orthogonal schedule embedded in the MAC. Reducing the broadcast latency by finding first critical-paths in a duty-cycled WSN and scheduling transmissions with a preference of nodes in the critical-path is proposed in [9]. 
Minimum Energy Multicasting with Adjusted Power (MEMAP) problem in the duty-cycled sensor networks is proposed in [10][11] by constructing approximate scheduling algorithm using an auxiliarygraph. Guard Beacon and beacon scheduling strategy are presented in [11][12] to reduce the overall power consumption of beacon sending and receiving. To maximize lifetime issue in WSNs by employing joint routing and asynchronous duty cycle scheduling techniques EB-JRADCS is introduced in [13].

Dynamic Switching-based Reliable Flooding (DSRF) framework is presented in [3] to provide efficient flooding in low-duty-cycle WSNs. DSRF dynamically adjust structure of flooding tree based on exploring poor links and good links on demand. DSRF is designed to address the problem of reliable flooding in unreliable communication links. It reduces flooding delay and energy cost by switching decision making based on a cost analysis when transmission failure.

In this paper, we introduce a new duty cycle algorithm based on Brain Storming Optimization (BSO). The algorithm proposed in this paper tries to minimize the energy consumed by the nodes through sending inactive nodes to sleep. The main idea is to define the best route for a period of time and nodes involoved in this route will be the only nodes active; others will be in their sleep mode. These nodes will be awake later periods if they got involved in the routing procedure.

The paper is organized as follows: section II explores the duty cycle problem in WSNs; section III briefly go over the Brain Storming Optimization Algorithm that is used in to solve the energy consumption problem proposed in this paper; the solution approach is presented in ection IV; simulation results are presented in section V while the paper concludes in section VI.

\section{DUTY CYCLE PROBLEM IN WSNS}

In WSNs, the main concern is the sensors and overall sensor network energy. Nodes may be deployment in unattended areas or in critical mission application in which the network may require the nodes to life for longer time. In order to solve such problem, nodes might need to be schedules between awake and sleep nodes. For this paper, we investigate the data reporting problem and how sensors can be scheduled to save their energy. This paper deals with two types of reporting which are source nodes reporting their data to the sink node and the sink node querying some of the source nodes.

Given a sensor network in a form of a graph $\mathrm{G}(\mathrm{V}, \mathrm{E})$ where $\mathrm{V}$ is the number of vertices (nodes) and $\mathrm{E}$ a set of links between the nodes. Each link $\mathrm{e} \in \mathrm{E}$ represents the communication between these two nodes. In other words, if two nodes are able to communicate with each other, this communication is represented by a link between them of the graph G. The sink node is assumed to be anywhere in the monitored field.

In a reporting WSN, we assume that there are a set of source nodes deployed in the monitored field. These source nodes sense the environment and send their data to the sink node. The sink node analyzes the received data and extracts the required monitoring information. On the other hand, the query WSN is based on the sink query to some/all of the source sensor nodes. For such types of networks, routing the data could be costly in terms of energy. Therefore, the main problem handled in this paper is to reliably route the data through the network and save its energy at the same time using duty cycle.

\section{III.BRAIN STORMING OPTIMIZATION (BSO)}

This is a brief description to the BSO algorithm that is used in our duty cycle problem; for more details on the BSO, the reader is referred to (Rabie and Ahmed 2016). This section proposes to apply a modified version of the Brain Storming Optimization (BSO) algorithm for the previously stated routing problem. The algorithm follows the real brain storming strategy footsteps and the adapted version of the algorithm can be described as follows:

Step 1: Define the number of iterations $\left(I_{\max }\right)$, number of ideas (D) to be initially generated,number of clusters $\left(\mathrm{K}_{\max }\right)$,

Step 2: Randomly generate D ideas.

Step 3: Evaluate the D generated ideas based on the coverage and consumed energy.

Step 4: Apply the Pareto dominance on the generated idea

Step 5: Apply K-Mean Clustering to cluster the generated ideas into number of clusters $K_{\max }$.

Step 6: Within each cluster, apply Crossover operation between the newly generated ideas and the old ones; select the best idea to replace the old one, if any.

Step 7: Rank the selected $\mathrm{X}$ solution from all clusters based on their coverage and lifetime and select the best no dominated ones (C_ideas).

Step 8: If the current number of iterations $\geq I_{\max }$, go to Step 10 .

Step 9: if the number of current ideas is less than D, randomly generate other ( D- C_ideas) ideas and go to step 3 .

Step 10: report the current idea and terminate. 
The crossover methodology used in this chapter inspired from [17]where three crossover methods are applied. The first crossover method is the random method where some of the sensors positions in each idea are exchange based on probability (Prob1). The second methodology is the MaxDist where sensors locations in each idea are adjusted to have the furthest distance between the two corresponding sensors in each idea; then the random crossover operator is used. The third crossover method is based on the MinDist normalization where sensors positions are rearranged to have the distance between sensors in corresponding ideas are minimized; then the random crossover operator is applied.

$\mathrm{K}$-means clustering is a famous clustering algorithm that tries to group objects based on their features into $\mathrm{K}$ classes where $\mathrm{k}$ is a positive number. The grouping is done by minimizing the distances between the data and the corresponding cluster centroid.

\section{IV.DUTY CYCLE APPROACH FOR WSNS ENERGY SAVING}

In this section we explain the main idea behind the duty cycle approach proposed in this paper for WSN energy saving. The duty cycle approach works in two ways; one of the methods is targeting the data reporting network and the second method is targeting the query based network.

For the data reporting network, sources, as stated, will be the main source of data. The main idea behind the duty cycle algorithm in this network is to select the best routes from the source nodes to the sink nodes that minimizes the network consumed energy. At the same time, by selecting the route for each source, other nodes not in the selected routes will go to sleep for certain period of time. On the other hand, for the query based networks, the sink node will identify the best routes to the sources and the source will be able to identify the best routes to the sink node according to the previous algorithm in the reporting network. There are two main criteria for anode to select the next node which are the residual energy, and number of times that have been selected in the routes in the previous rounds. Each node, will select the next node to reach the sink node or vice versa. It is expected to have some of the common nodes between the different sources or in the paths between the sink node and the queried sources.

\section{SIMULATION EXPERIMENTS}

Here again as stated in our previous work[18], we base our work on the first-order model described in[16] where the transmitter or the receiver dissipates $\mathrm{E}_{\text {elec }}$ energy per bit to run the digital coding circuit, modulation circuit, and filtering of the signal circuit which are the radio electronic circuits before it is sent to the amplifier and dissipates $\mathrm{E}_{\mathrm{amp}}$ in the power amplifier as shown in Figure 1. $E_{\text {amp }}$ Varies according to the distance $d$ between a transmitter and a receiver: $\mathrm{E}_{\mathrm{amp}}=\varepsilon_{\mathrm{fs}}$ assuming a free space model when $d i s<\mathrm{d}_{\circ}$ and $\alpha=2$, while $\mathrm{E}_{\mathrm{amp}}=\varepsilon_{\mathrm{mp}}$ assuming a multi-path model when $d_{i s} \geq \mathrm{d}_{\circ}$ and $\alpha=4$, where $\left.\mathrm{d}_{-} \circ=\sqrt{\left(2 \& \varepsilon_{-}\right.} \mathrm{fs}_{\mathrm{s}} / \varepsilon_{-} \mathrm{mp}\right)$ Thus, to transmit an $\ell b i t$-bit packet over distance dis, the radio expends to:

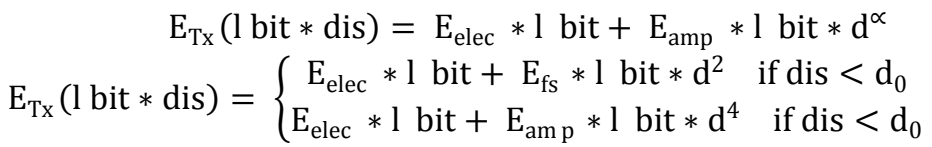

and to receive this message, the radio expends:

$$
\mathrm{E}_{\mathrm{TX}}(1 \mathrm{bit} * \text { dis })=\mathrm{E}_{\text {elec }} * 1 \text { bit }
$$

In these formulas, $\mathrm{E}_{\mathrm{Tx}}$ is the transmission power, $\mathrm{E}_{\mathrm{Rx}}$ is the receiver power. The radios are assumed to have power control and consume the minimal energy needed to reach the receiver. The monitored area is assumed $800 \mathrm{mX} 800 \mathrm{~m}$ and the number of used sensors are assumed 100 . The 100 sensors are assumed to be from three different categories in which they differ in their sensing ranges, communication ranges, and initial energy. The first category involves sensors with sensing range from $30 \mathrm{~m}$, communication range of $60 \mathrm{~m}$, and initial energy $0.5 \mathrm{j}$. The second category includes $50 \mathrm{~m}$ sensing range, $100 \mathrm{~m}$ communication range, and $1 \mathrm{j}$ as initial energy. The sensing range of the last category is $60 \mathrm{~m}$ and the communication range is $90 \mathrm{~m}$ and the initial energy is assumed to be $1.5 \mathrm{j}$. The number of each category is selected based on a probability $\mathrm{P}($ type) where each experiment runs for 10 times with different settings and different number of sensors per category; the average over the 10 runs are reported in the following case studies. A common energy model is assumed for all of them as can be seen in TABLE IERROR! REFERENCE SOURCE NOT FOUND.

TABLE II COMMON ENERGY MODEL

\begin{tabular}{|l|l|}
\hline $\mathrm{E}_{\text {elec }}=\mathrm{E}_{\mathrm{DA}}$ & $50 \mathrm{~nJ} / \mathrm{bit}$ \\
\hline $\mathrm{E}_{\mathrm{fs}}$ & $10 \mathrm{pJ} / \mathrm{bit} / \mathrm{m}^{2}$ \\
\hline $\mathrm{E}_{\mathrm{mp}}$ & $0.0013 \mathrm{pJ} / \mathrm{bit}^{2} \mathrm{~m}^{4}$ \\
\hline
\end{tabular}


In the following set of experiments, we examine the performance of the BSO algorithm in terms of energy saving compared to non-duty cycle algorithm. In non-duty cycle algorithm, we define the routes but we do not allow nodes to go to sleep. We divide these set of experiments into three categories; the first category examines the network energy savings when homogenous sensors are utilized and the second category tests the network performance when heterogeneous set of sensors are deployed in the monitored field. The third category tries to show the performance of the BSO with increasing the number of sensors in the monitored field.

As can be seen in Figure 1, 200 nodes are deployed uniformly in the monitored field; these nodes are having the same characteristics in terms of energy, memory, sensing, communication, and processing capabilities. 10000 events are generated in the monitored field and sensed by the sensors.

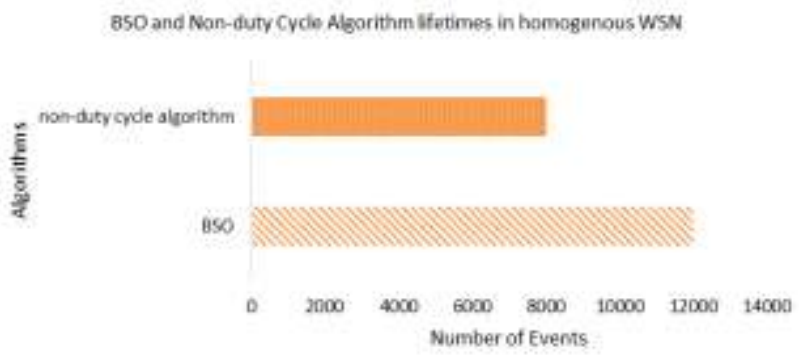

Figure 1: Performance of BSO algorithms in homogenous network

Figure 1 shows the comparison between the proposed BSO and the Non-duty cycle algorithm in terms of the network lifetime. The network lifetime is measured using the survival of the networks with the number of detected events. BSO was able to survive to detect all of the generated 10000 events while Non-duty cycle algorithm died after 6000 event. A similar set of experiments is done using heterogeneous WSNs as shown in Figure 2. As can be seen, BSO was able to survive to detect 12000 events from the monitored field while the Non-duty cycle network was able to survive to detect only 8000 events. This is also indicating heterogeneous networks might be better than homogenous networks in terms of WSNs lifetime.

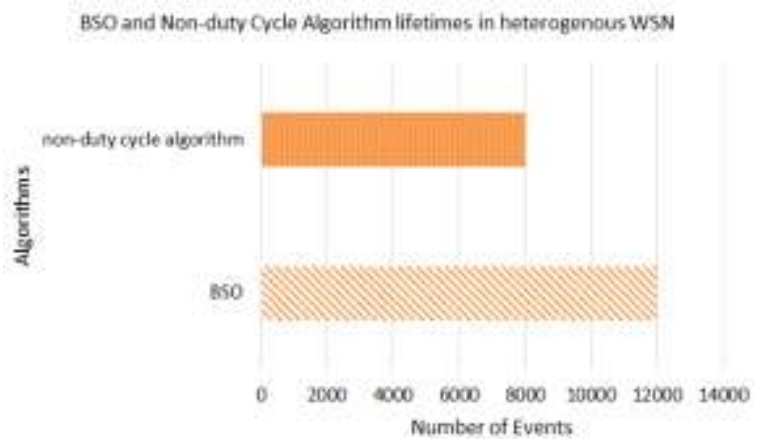

Figure 2: Performance of $\mathrm{BSO}$ algorithms in heterogeneous network

Figure 3 shows another set of experiments where the BSO is examined with different number of nodes. Again, it seems that BSO is outperforming the Non-duty cycle algorithm in terms of energy saving in all networks and settings. One of the notable things in Figure 3 is that as the number of nodes increases, it seems that lifetime difference of the network represented in the number of detected events between the BSO and the Non-duty cycle algorithm is not linear where BSO is performing much better than Non-duty cycle algorithm.

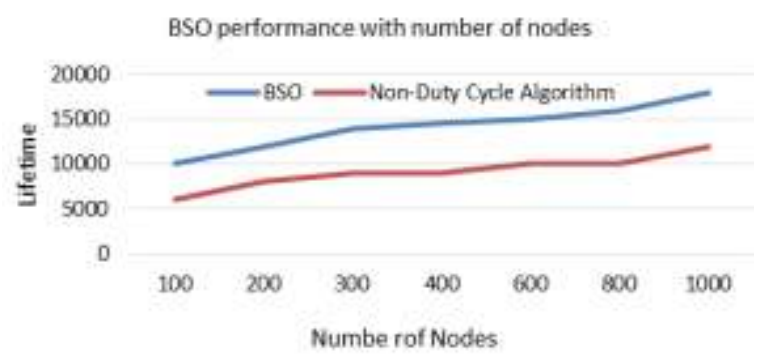

Figure 3: Performance of $\mathrm{BSO}$ algorithms with increasing the number of heterogeneous sensors 


\section{VI.CONCLUSION}

In this paper, we introduced a new proposed method for energy saving in WSNs. This method utilizes the BSO inspired from human thinking to solve one of the difficult problems which is routing with nodes duty cycles. The idea of the proposed BSO algorithm is to let the nodes that are not participating in the routing go to sleep. These nodes will be awake after certain period to be chosen in the next routing interval. The algorithm is extensively compared to Nonduty cycle algorithm where nodes will never go to sleep. The experiments are done on two types of networks which are homogenous and heterogeneous networks. The results show that BSO is outperforming the Non-duty cycle algorithm

\section{REFERENCES}

[1] Kai Han, Jun Luo, Yang Liu, andAthanasios V. Vasilakos, "Algorithm design for data communications in duty-cycled wireless sensor networks: A survey”, IEEE Communications Magazine, pp 107-113, July 2013

[2] Nauman Mushtaq, Shagufta Henna, “Asynchronous Hybrid Duty Cycle MAC Protocol for Wireless Sensor Networks”, International Journal of Computer Applications (0975 - 8887) Volume 131 - No.3, Dec. 2015.

[3] Long Cheng, Jianwei Niu, Senior, Yu Gu, Chengwen Luo, and Tian He, “Achieving Efficient Reliable Flooding in Low-Duty-Cycle Wireless Sensor Networks", IEEE/ACM Transactions on Networking, vol. 24, no. 6, pp. 3676-3689, DEC. 2016.

[4] Shaobo Wu, Jianwei Niu, Wusheng Chou, and Mohsen Guizani, "Delay-Aware Energy Optimization for Flooding in Duty-Cycled Wireless Sensor Networks", IEEE Transactions on Wireless Communications, Vol 15, Issue 12, pp: 8449 - 8462, Dec. 2016.

[5] Shuguang Xiong, Jianzhong Li, Mo Li, Jiliang Wang, and Yunhao Liu, "Multiple task scheduling for low-duty-cycled wireless sensor networks", Proceedings IEEE INFOCOM, April 10-15, Shanghai, P.R. China, pp. 1323-1331, 2011.

[6] Chunsheng Zhu, Laurence T. Yang, Lei Shu, Joel J. P. C. Rodrigues, and Takahiro Hara, “A Geographic Routing Oriented Sleep Scheduling Algorithm in Duty-Cycled Sensor Networks", IEEE ICC 2012 - Wireless Networks Symposium, pp. 5473- 5477, 2012.

[7] Duc Tai Le and Thang Le Duc, Vyacheslav V. Zalyubovskiy, and Hyunseung Choo, "Latency Aware Broadcast Scheduling in Duty Cycled Wireless Sensor Networks", The International Conference on Information Networking (ICOIN 2013), 28-30 Jan. 2013, Bangkok, Thailand

[8] Jian Lin and Mary Ann Ingram, "OSC-MAC: Duty Cycle Scheduling and Cooperation in Multi-hop Wireless Sensor Networks", IEEE Wireless Communications and Networking Conference (WCNC 2013): MAC., Shanghai, China, 2013.

[9] Giyeol Im, Duc Tai Le, Hyunseung Choo, and Dongsoo S. Kim, "Critical-path aware broadcast scheduling in duty-cycled Wireless Sensor Networks", International Conference on Information Networking (ICOIN 2015), 12-14 Jan. 2015, Siem Reap, Cambodia.

[10] Quan Chen, Hong Gao, Siyao Cheng, and Zhipeng Cai "Approximate Scheduling and Constructing Algorithms for Minimum-Energy Multicasting in Duty-Cycled Sensor Networks", International Conference on Identification, Information, and Knowledge in the Internet of Things, 22-23 Oct. 2015, Beijing, China, 2015.

[11] Chen Q., Cheng S., Gao H., Li J., Cai Z. Energy-Efficient Algorithm for Multicasting in Duty-Cycled Sensor Networks. Sensors. 2015;15:31224-31243

[12] Yongrui Chen, Yulong Xing, and Weidong Yi, "Optimal Beacon Scheduling for Low-duty-cycle Sensor Networks", IEEE International Conference on Communications (ICC 2016), 22-27 May 2016, Kuala Lumpur, Malaysia.

[13] Mohsen Kariman-Khorasani, Mohammad Ali Pourmina, Ahmad Salahi, "Energy Balance Based Lifetime Maximization in Wireless Sensor Networks Employing Joint Routing and Asynchronous Duty Cycle Scheduling Techniques", Wireless Personal Communications, July 2015, Volume 83, Issue 2, pp 1057-1083

[14] Ha, Nguyen Phan Khanh; Zalyubovskiy, Vyacheslav; Choo, Hyunseung, "Design a Delay-Efficient Data Aggregation Scheduling Scheme for Duty-cycled Wireless Sensor Networks", International Information Institute (Tokyo). Information; Koganei, vol 16, no 8, pp 5755-5771,Aug 2013.

[15] Shiliang Xiao, Jingchang Huang, Lebing Pan, Yongbo Cheng, and Jianpo Liu, "On centralized and distributed algorithms for minimizing data aggregation time in duty-cycled wireless sensor networks”, Wireless Networks, Oct. 2014, Vol 20, Issue 7, pp 1729-1741.

[16] Ramalakshmi, R. \& Radhakrishnan, S. 2015. Connected k-Coverage Topology Control for Area Monitoring in Wireless Sensor Networks. Wireless Personal Communications, 84(2): 1051-1067

[17] Junfeng, C. Shi, C. Yang, C., Yingjuan, X. Yuhui, S. 2015. Enhanced Brain Storm Optimization Algorithm for Wireless Sensor Networks Deployment,Advances in Swarm and Computational Intelligence.

[18] Rabie A. Ramadan, Ahmed Y. Khder, "Brain Storming Algorithm for Coverage and Connectivity Problem in Wireless Sensor Networks," ICCMIT'16, 2016.

\section{BIOGRAPHY}

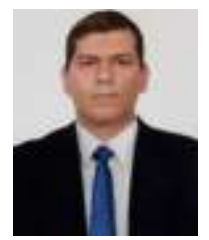

Ahmed Y. Khedr is an Assistant professor in the Department of System and Computer Engineering in Al-Azhar University, Cairo, Egypt. Ahmed is working now in Computer Science and Engineering in Hail University, Hail, Saudi Arabia. Ahmed was funded by the Egyptian government to visit SMU at USA and conduct research in Mobile Computing with the PDA Mobile research group. Ahmed's research area is focused on wireless sensor networks, data mining, and e-learning algorithms. 